\title{
Search for an Excess of Events in the Super-Kamiokande Detector in the Directions of the Astrophysical Neutrinos Reported by the IceCube Collaboration
}

K. Abe ${ }^{1,2}$, C. Bronner ${ }^{1}$, G. Pronost ${ }^{1}$, Y. Hayato ${ }^{1,2}$, M. Ikeda ${ }^{1}$, K. Iyogi ${ }^{1}$, J. Kameda ${ }^{1,2}$, Y. Kato ${ }^{1}$, Y. Kishimoto ${ }^{1,2}$, Ll. Marti ${ }^{1}$, M. Miura ${ }^{1}$, S. Moriyama ${ }^{1}$, M. Nakahata ${ }^{1,2}$, Y. Nakano ${ }^{1}$, S. Nakayama ${ }^{1,2}$, Y. Okajima ${ }^{1}$, A. Orii ${ }^{1}$, H. Sekiya ${ }^{1,2}$, M. Shiozawa ${ }^{1,2}$, Y. Sonoda ${ }^{1}$, A. Takeda ${ }^{1,2}$, A. Takenaka ${ }^{1}$, H. Tanaka ${ }^{1}$, S. Tasaka ${ }^{1}$, T. Tomura ${ }^{1,2}$, R. Akutsu ${ }^{3}$, T. Kajita ${ }^{2,3}$, K. Kaneyuki ${ }^{2,3,46}$, Y. Nishimura ${ }^{3}$, K. Okumura ${ }^{2,3}$, K. M. Tsui ${ }^{3}$, L. Labarga ${ }^{4}$, P. Fernandez ${ }^{4}$, F. d. M. Blaszczyk ${ }^{5}$, J. Gustafson ${ }^{5}$, C. Kachulis ${ }^{5}$, E. Kearns ${ }^{2,5}$, J. L. Raaf ${ }^{5}$, J. L. Stone ${ }^{2,5}$, L. R. Sulak ${ }^{5}$, S. Berkman ${ }^{6}$, S. Tobayama ${ }^{6}$, M. Goldhaber ${ }^{7,46}$, M. Elnimr ${ }^{8}$, W. R. Kropp ${ }^{8}$, S. Mine ${ }^{8}$, S. Locke ${ }^{8}$, P. Weatherly ${ }^{8}$, M. B. Smy ${ }^{2,8}$, H. W. Sobel ${ }^{2,8}$, V. Takhistov ${ }^{8,47}$, K. S. Ganezer ${ }^{9}$, J. Hill ${ }^{9}$, J. Y. Kim ${ }^{10}$,

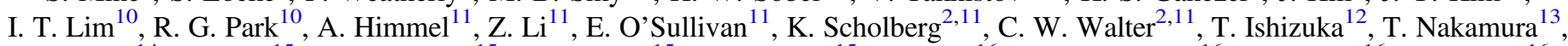
J. S. Jang ${ }^{14}$, K. Choi ${ }^{15}$, J. G. Learned ${ }^{15}$, S. Matsuno ${ }^{15}$, S. N. Smith ${ }^{15}$, J. Amey ${ }^{16}$, R. P. Litchfield ${ }^{16}$, W. Y. Ma ${ }^{16}$, Y. Uchida ${ }^{16}$, M. O. Wascko ${ }^{16}$, S. Cao ${ }^{17}$, M. Friend ${ }^{17}$, T. Hasegawa ${ }^{17}$, T. Ishida ${ }^{17}$, T. Ishii ${ }^{17}$, T. Kobayashi ${ }^{17}$, T. Nakadaira ${ }^{17}$, K. Nakamura ${ }^{2,17}$, Y. Oyama ${ }^{17}$, K. Sakashita ${ }^{17}$, T. Sekiguchi ${ }^{17}$, T. Tsukamoto ${ }^{17}$, KE. Abe ${ }^{18}$, M. Hasegawa ${ }^{18}$, A. T. Suzuki ${ }^{18}$, Y. Takeuchi ${ }^{2,18}$, T. Yano ${ }^{18}$, S. V. Cao ${ }^{19}$, T. Hayashino ${ }^{19}$, T. Hiraki ${ }^{19}$, S. Hirota ${ }^{19}$, K. Huang ${ }^{19}$, M. Jiang ${ }^{19}$, A. Minamino ${ }^{19}$, KE. Nakamura ${ }^{19}$, T. Nakaya ${ }^{2,19}$, B. Quilain ${ }^{19}$, N. D. Patel ${ }^{19}$, R. A. Wendell ${ }^{2,19}$, L. H. V. Anthony ${ }^{20}$, N. McCauley ${ }^{20}$, A. Pritchard ${ }^{20}$, Y. Fukuda ${ }^{21}$, Y. Itow $^{22,23}$ (1), M. Murase ${ }^{22}$, F. Muto ${ }^{22}$, P. Mijakowski ${ }^{24}$, K. Frankiewicz ${ }^{24}$, C. K. Jung ${ }^{25}$, X. Li ${ }^{25}$, J. L. Palomino ${ }^{25}$, G. Santucci ${ }^{25}$, C. Vilela ${ }^{25}$, M. J. Wilking ${ }^{25}$, C. Yanagisawa ${ }^{25,48}$, S. Ito $^{26}$, D. Fukuda ${ }^{26}$, H. Ishino ${ }^{26}$, A. Kibayashi ${ }^{26}$, Y. Koshio ${ }^{2,26}$ (1) H. Nagata ${ }^{26}$, M. Sakuda ${ }^{26}$, C. Xu ${ }^{26}$, Y. Kuno ${ }^{27}$, D. Wark ${ }^{28,29}$, F. Di Lodovico ${ }^{30}$, B. Richards ${ }^{30}$, R. Tacik ${ }^{31,32}$, S. B. Kim ${ }^{33}$, A. Cole ${ }^{34}$, L. Thompson ${ }^{34}$, H. Okazawa ${ }^{35}$, Y. Choi ${ }^{36}$, K. Ito ${ }^{37}$, K. Nishijima ${ }^{37}$, M. Koshiba ${ }^{38}$, Y. Totsuka ${ }^{38,46}$, Y. Suda ${ }^{39}$, M. Yokoyama ${ }^{2,39}$, R. G. Calland ${ }^{2}$, M. Hartz ${ }^{2}$, K. Martens ${ }^{2}$, C. Simpson ${ }^{2,28}$, Y. Suzuki ${ }^{2}$, M. R. Vagins ${ }^{2,8}$, D. Hamabe ${ }^{40}$, M. Kuze ${ }^{40}$, T. Yoshida ${ }^{40}$, M. Ishitsuka ${ }^{41}$, J. F. Martin ${ }^{42}$, C. M. Nantais ${ }^{42}$, H. A. Tanaka ${ }^{42}$, A. Konaka ${ }^{32}$, S. Chen ${ }^{43}$, L. Wan ${ }^{43}$, Y. Zhang ${ }^{43}$, A. Minamino ${ }^{44}$, and R. J. Wilkes ${ }^{45}$

The Super-Kamiokande Collaboration

\footnotetext{
${ }^{1}$ Kamioka Observatory, Institute for Cosmic Ray Research, University of Tokyo, Kamioka, Gifu 506-1205, Japan

${ }^{2}$ Kavli Institute for the Physics and Mathematics of the Universe (WPI), The University of Tokyo Institutes for Advanced Study, University of Tokyo, Kashiwa, Chiba 277-8583, Japan

${ }^{3}$ Research Center for Cosmic Neutrinos, Institute for Cosmic Ray Research, University of Tokyo, Kashiwa, Chiba 277-8582, Japan

${ }^{4}$ Department of Theoretical Physics, University Autonoma Madrid, E-28049 Madrid, Spain

${ }^{5}$ Department of Physics, Boston University, Boston, MA 02215, USA

${ }^{6}$ Department of Physics and Astronomy, University of British Columbia, Vancouver, BC V6T 1Z4, Canada

${ }^{7}$ Physics Department, Brookhaven National Laboratory, Upton, NY 11973, USA

${ }^{8}$ Department of Physics and Astronomy, University of California, Irvine, Irvine, CA 92697-4575, USA

Department of Physics, California State University, Dominguez Hills, Carson, CA 90747, USA

${ }^{10}$ Department of Physics, Chonnam National University, Kwangju 500-757, Korea

${ }^{11}$ Department of Physics, Duke University, Durham NC 27708, USA

12 Junior College, Fukuoka Institute of Technology, Fukuoka, Fukuoka 811-0295, Japan

${ }^{13}$ Department of Physics, Gifu University, Gifu, Gifu 501-1193, Japan

${ }^{14}$ GIST College, Gwangju Institute of Science and Technology, Gwangju 500-712, Korea

${ }^{15}$ Department of Physics and Astronomy, University of Hawaii, Honolulu, HI 96822, USA

${ }^{16}$ Department of Physics, Imperial College London, London SW7 2AZ, UK

${ }^{17}$ High Energy Accelerator Research Organization (KEK), Tsukuba, Ibaraki 305-0801, Japan

${ }_{19}^{18}$ Department of Physics, Kobe University, Kobe, Hyogo 657-8501, Japan

${ }^{19}$ Department of Physics, Kyoto University, Kyoto, Kyoto 606-8502, Japan

${ }^{20}$ Department of Physics, University of Liverpool, Liverpool L69 7ZE, UK

${ }^{21}$ Department of Physics, Miyagi University of Education, Sendai, Miyagi 980-0845, Japan

${ }^{22}$ Institute for Space-Earth Environmental Research, Nagoya University, Nagoya, Aichi 464-8602, Japan

${ }^{23}$ Kobayashi-Maskawa Institute for the Origin of Particles and the Universe, Nagoya University, Nagoya, Aichi 464-8602, Japan ${ }^{24}$ National Centre For Nuclear Research, 00-681 Warsaw, Poland

${ }^{25}$ Department of Physics and Astronomy, State University of New York at Stony Brook, Stony Brook, NY 11794-3800, USA

${ }^{26}$ Department of Physics, Okayama University, Okayama, Okayama 700-8530, Japan

${ }^{27}$ Department of Physics, Osaka University, Toyonaka, Osaka 560-0043, Japan ${ }^{28}$ Department of Physics, Oxford University, Oxford OX1 3PU, UK

${ }^{29}$ STFC, Rutherford Appleton Laboratory, Harwell Oxford, and Daresbury Laboratory, Warrington OX11 0QX, UK

${ }^{30}$ School of Physics and Astronomy, Queen Mary University of London, London E1 4NS, UK

${ }^{31}$ Department of Physics, University of Regina, 3737 Wascana Parkway, Regina, SK S4S OA2, Canada

${ }^{32}$ TRIUMF, 4004 Wesbrook Mall, Vancouver, BC V6T 2A3, Canada

${ }^{33}$ Department of Physics, Seoul National University, Seoul 151-742, Korea

${ }^{34}$ Department of Physics and Astronomy, University of Sheffield, S10 2TN, Sheffield, UK

${ }^{35}$ Department of Informatics in Social Welfare, Shizuoka University of Welfare, Yaizu, Shizuoka 425-8611, Japan

${ }^{36}$ Department of Physics, Sungkyunkwan University, Suwon 440-746, Korea

${ }^{37}$ Department of Physics, Tokai University, Hiratsuka, Kanagawa 259-1292, Japan ${ }^{38}$ The University of Tokyo, Bunkyo, Tokyo 113-0033, Japan

${ }^{39}$ Department of Physics, University of Tokyo, Bunkyo, Tokyo 113-0033, Japan

${ }^{40}$ Department of Physics, Tokyo Institute of Technology, Meguro, Tokyo 152-8551, Japan

${ }^{41}$ Department of Physics, Faculty of Science and Technology, Tokyo University of Science, Noda, Chiba 278-8510, Japan
} 


$$
\begin{gathered}
{ }^{42} \text { Department of Physics, University of Toronto, ON M5S 1A7, Canada } \\
{ }^{43} \text { Department of Engineering Physics, Tsinghua University, Beijing, 100084, China } \\
{ }^{44} \text { Faculty of Engineering, Yokohama National University, Yokohama, 240-8501, Japan } \\
{ }^{45} \text { Department of Physics, University of Washington, Seattle, WA 98195-1560, USA } \\
\text { Received } 2017 \text { July 25; revised } 2017 \text { October 18; accepted 2017 October 18; published 2017 November } 28
\end{gathered}
$$

\begin{abstract}
We present the results of a search in the Super-Kamiokande (SK) detector for excesses of neutrinos with energies above a few $\mathrm{GeV}$ that are in the direction of the track events reported in IceCube. Data from all SK phases (SK-I through SK-IV) were used, spanning a period from 1996 April to 2016 April and corresponding to an exposure of 225 kiloton-years. We considered the 14 IceCube track events from a data set with 1347 livetime days taken from 2010 to 2014. We use Poisson counting to determine if there is an excess of neutrinos detected in SK in a $10^{\circ}$ search cone $\left(5^{\circ}\right.$ for the highest energy data set) around the reconstructed direction of the IceCube event. No significant excess was found in any of the search directions we examined. We also looked for coincidences with a recently reported IceCube multiplet event. No events were detected within a $\pm 500 \mathrm{~s}$ time window around the first detected event, and no significant excess was seen from that direction over the lifetime of SK.
\end{abstract}

Key words: astroparticle physics - neutrinos

\section{Introduction}

Neutrino astronomy is a burgeoning field, bridging the gap between astronomy and particle physics. Neutrinos travel undistorted from their source and are therefore a valuable probe of the inner workings of astrophysical phenomena. The first definitive measurement of high-energy extragalactic neutrinos was made by the IceCube experiment in 2013 (IceCube Collaboration 2013), where they were able to reject the atmospheric-neutrino-only hypothesis at greater than $4 \sigma$.

Detecting neutrinos that are astrophysical in origin has raised many questions. Where are these neutrinos coming from? What process is creating them? There has not yet been any significant evidence to suggest that these neutrinos are pointing to a particular region of the sky. The many searches for counterparts to the neutrino signal have been largely unsuccessful, including searches for coincidences with photons from Fermi-LAT (Peng \& Wang 2017), fast radio bursts (Fahey et al. 2016), as well as a search for coincidences with a large catalog of candidate sources (Aartsen et al. 2017). One search (Kadler et al. 2016) found a coincidence between a PeV neutrino detected in IceCube and an outburst of the blazar PKS B1424418, giving a hint at a possible origin of these astrophysical neutrinos.

IceCube uses detected events with energies above a few hundred $\mathrm{TeV}$ to look for astrophysical neutrinos. In this energy region, the atmospheric neutrino background is expected to be low. However, now that astrophysical neutrino candidates have been identified, one can search in the lower-energy data for an excess of events coming from the same direction. Given the unknown origin of these neutrinos, searching this previously unexplored energy region is of interest.

Super-Kamiokande (SK), a water Cherenkov detector located in Japan, detects atmospheric neutrinos in the energy range of $30 \mathrm{MeV}$ to several $\mathrm{TeV}$. For events with energies above a few $\mathrm{GeV}$, the direction of the incoming neutrino can be reconstructed as it is well correlated with the direction of the detected outgoing lepton.

SK has performed a number of astrophysical neutrino searches in the past. Recent searches include a general, all-

\footnotetext{
46 Deceased.

47 Also at Department of Physics and Astronomy, UCLA, CA 90095-1547, USA.

48 Also at BMCC/CUNY, Science Department, New York, NY, USA.
}

sky astrophysical search (Abe et al. 2006; Swanson et al. 2006; Thrane et al. 2009b); searches for coincidences with gammaray bursts, supernova remnants, and other potential sources of astrophysical neutrinos (Desai et al. 2008; Thrane et al. 2009a, 2009b); dark matter searches (Tanaka et al. 2011; Choi et al. 2015); and a search for coincidences with the recent detection of gravitational-wave signals (Abe et al. 2016).

In this paper, we look for excesses of neutrino events in Super-Kamiokande in the direction of the IceCube events from their data release in The IceCube Collaboration (2015). We use the IceCube events that have the best pointing accuracy, known as track events, and determine if there is an excess of events in the full SK high-energy data set. Given the uncertainty about the origins of these astrophysical neutrinos, we perform a model-blind search, without assuming an energy spectrum a priori. Since we have no observational or theoretical motivation for the time duration over which these neutrinos are emitted, we do not require any timing correlation between the IceCube and SK events. For this simple estimate, we omit discussions of systematic errors. We also report the search for coincidences with the recent multiplet event reported in Aartsen et al. (2017).

An estimate of the number of events anticipated in the SK sample can be derived by extending the point-source limits from Aartsen et al. (2016) down to lower energies. Assuming the standard $E^{-2}$ spectrum, we would expect approximately 0.5 events in the UPMU sample and approximately 0.002 events in fully contained (FC) and partially contained (PC) samples. If we assume a cutoff of $E_{\nu}<100 \mathrm{TeV}$ (which is below the deposited energies of IceCube events 4, 9, and 12), we would expect around 5 events in the UPMU sample and around 0.02 events in the FC and PC samples. More events could be expected in the SK topologies if there is a softer spectral index (in The IceCube Collaboration 2015 the best-fit index is $-2.58 \pm 0.25$ ) or if there is a broken power law between the higher-energy domain described by IceCube and the lower energies seen in SK.

\section{The Super-Kamiokande Experiment}

The SK detector is a 50 kilotonne (22.5 kilotonne fiducial) water Cherenkov detector located in the Mozumi mine in the Gifu prefecture of Japan. The cylindrical detector is optically separated into an inner detector (ID) volume, which is viewed 
by $\sim 11,000$ photomultiplier tubes (PMTs), and an outer detector (OD) volume, which is viewed by $\sim 2000$ PMTs. A more detailed description of the SK detector can be found in Fukuda et al. (2003).

The SK data are divided into four experimental phases. SK-I ran from 1996 to 2001 with $40 \%$ photocoverage. In 2001, there was an accidental implosion that damaged some of the PMTs. SK-II ran from 2002 to 2005 with a photocoverage of $20 \%$. In 2006, the damaged phototubes were repaired and the SK-III phase began with $40 \%$ photocoverage. After an electronics upgrade in 2008, the current phase of the experiment, SK-IV, began. Data from all four phases of the experiment are used in this analysis, spanning 1996 April to 2016 April and corresponding to 225 kilotonne-years.

Detected neutrino events at energies above $30 \mathrm{MeV}$ can have three different topologies in the SK detector. The first, known as FC events, have a reconstructed vertex inside the fiducial volume, with little light seen in the OD. Events that have a reconstructed vertex inside the fiducial volume, but have interaction products that produce light in the OD, are known as PC events. Finally, muon neutrinos that interact in the surrounding rock and produce penetrating muons are known as upward-going muon (UPMU) events. We require these events to be coming from below the horizon in order to distinguish them from cosmic muons. These topologies roughly represent increasing, though overlapping, energy regions. For the atmospheric neutrino energy spectrum, FC events have an average energy of about $1 \mathrm{GeV}$, PC events have an average energy of about $10 \mathrm{GeV}$, and UPMU events have an average energy of about $100 \mathrm{GeV}$. More information on the SK topologies, including the selection cuts used in the data reduction, can be found in Ashie et al. (2005).

\section{Search Method}

The IceCube search directions were taken from the 2015 October data release (The IceCube Collaboration 2015). This data set contains neutrino candidates with two different topologies: track and shower. Track events are mainly from muon neutrino charged-current interactions, while shower events are mainly from neutral current interactions, as well as electron and tau neutrino charged-current interactions. Only IceCube track events were used in this analysis. Shower events typically have poor angular resolution, with an error up to $20^{\circ}$ for the (The IceCube Collaboration 2015) data set, making them unsuitable for a coincidence analysis using only spatial information. Track events, on the other hand, have a median angular resolution of better than $1^{\circ}$, allowing us to perform a spatial coincident search. Table 1 lists the properties of the IceCube events.

To ensure that the detected lepton points back to the incoming neutrino, a low energy threshold was imposed on the FC and PC data sets. A minimum energy requirement was determined by calculating the lowest energy such that $50 \%$ of the reconstructed lepton directions of that energy agreed to within $10^{\circ}$ of the incoming neutrino direction in the simulated data set from our Monte Carlo (MC) code. This threshold was determined to be $3.8 \mathrm{GeV}$ for FC events and $2.1 \mathrm{GeV}$ for PC events. No explicit upper energy cut was applied.

A search cone, centered at the reconstructed direction of each IceCube event, was defined with a half-angle opening of $10^{\circ}$ for FC and PC events and $5^{\circ}$ for UPMU events. The UPMU events are higher in energy than the other topologies and
Table 1

Information on the Track Events from IceCube Used in This Coincidence Search

\begin{tabular}{lccc}
\hline \hline Event Number & Decl. (degrees) & R.A. (degrees) & $\begin{array}{c}\text { Deposited } \\
\text { Energy (TeV) }\end{array}$ \\
\hline 1 & -31.2 & 127.9 & 78.7 \\
2 & -0.4 & 110.6 & 71.4 \\
3 & -21.2 & 182.4 & 32.6 \\
4 & 40.3 & 67.9 & 252.7 \\
5 & -24.8 & 345.6 & 31.5 \\
6 & -13.2 & 208.7 & 82.2 \\
7 & -71.5 & 164.8 & 46.1 \\
8 & 20.7 & 167.3 & 30.8 \\
9 & 14.0 & 93.3 & 200.5 \\
10 & -22.0 & 206.6 & 46.5 \\
11 & 0.0 & 336.7 & 84.6 \\
12 & -86.3 & 219.0 & 429.9 \\
13 & 67.4 & 209.4 & 74.3 \\
14 & -37.7 & 239.0 & 27.6 \\
\hline
\end{tabular}

Note. The data were taken from The IceCube Collaboration (2015).

therefore the detected lepton points back to the incoming neutrino with more accuracy, allowing for a smaller search cone.

In this analysis, only basic selection cuts were applied postreduction. For the FC data set, the cuts ensured that the reconstructed event vertex was more than $2.0 \mathrm{~m}$ from the detector wall, that the visible energy in the detector was greater than $3.8 \mathrm{GeV}$, and that there were fewer than 16 hits (10 for SK-I) in the outer detector volume. For the PC data set, the cuts ensured that the reconstructed event vertex was more than $2.0 \mathrm{~m}$ from the detector wall, that the visible energy in the detector was greater than $2.1 \mathrm{GeV}$, and that there was greater than or equal to 16 hits (10 for SK-I) in the outer detector volume. Finally, the UPMU data selection cuts ensured that the fit direction was below the horizon, that the fit track length was greater than $7.0 \mathrm{~m}$ for the events that the fitter classified as passing through the detector, and that the fitted momentum of the lepton was greater than $1.6 \mathrm{GeV}$ for events that the fitter classified as stopping in the detector.

To determine if there is an excess of events coming from the direction of the high-energy IceCube track events, Poisson statistics were used. A test statistic was constructed based on the maximum likelihood method. The likelihood of seeing $N$ events in our search cone given the expected number of background events including oscillations $\left(N_{B}\right)$ is

$$
L=\frac{e^{\frac{-N_{B}}{(1-\alpha)}}}{N !}\left(\frac{N_{B}}{1-\alpha}\right)^{N}
$$

where $\alpha$ represents the fraction of the $N$ events that are from the signal and is the parameter over which we maximize.

The background to this search is from atmospheric neutrinos. The SK MC code, along with a scaling for neutrino oscillations and the overall normalization of the flux, was used to determine the number of background events $\left(N_{B}\right)$ we expect to see in our search cone. The MC code used Geant3 (Brun et al. 1987) to simulate particle interactions and tracking. We used 500 years of simulated atmospheric neutrino events for each SK phase. The truth information was generated using nuclear interaction models used in NEUT (Hayato 2009). We scaled the MC sample for each phase to the appropriate livetime, and then we 

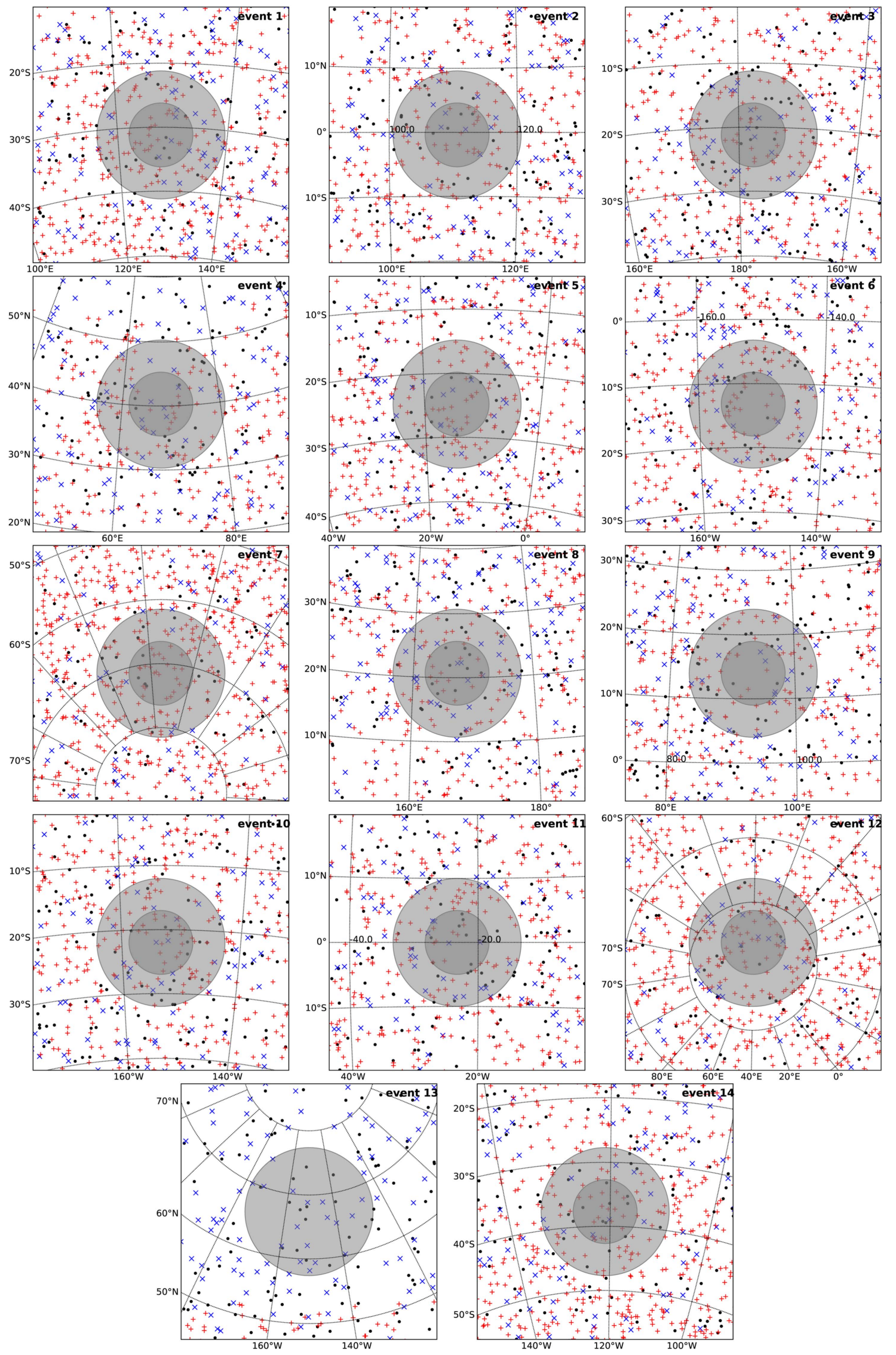

Figure 1. Detected FC (black circle), PC (blue cross), and UPMU (red plus) events in and around the search window. The position of events are shown in equatorial coordinates with right ascension on the $x$-axis and declination on the $y$-axis. The dark gray disk shows the $5^{\circ}$ search cone used for UPMU events, while the light gray disk shows the $10^{\circ}$ search cone used for FC and PC events. 

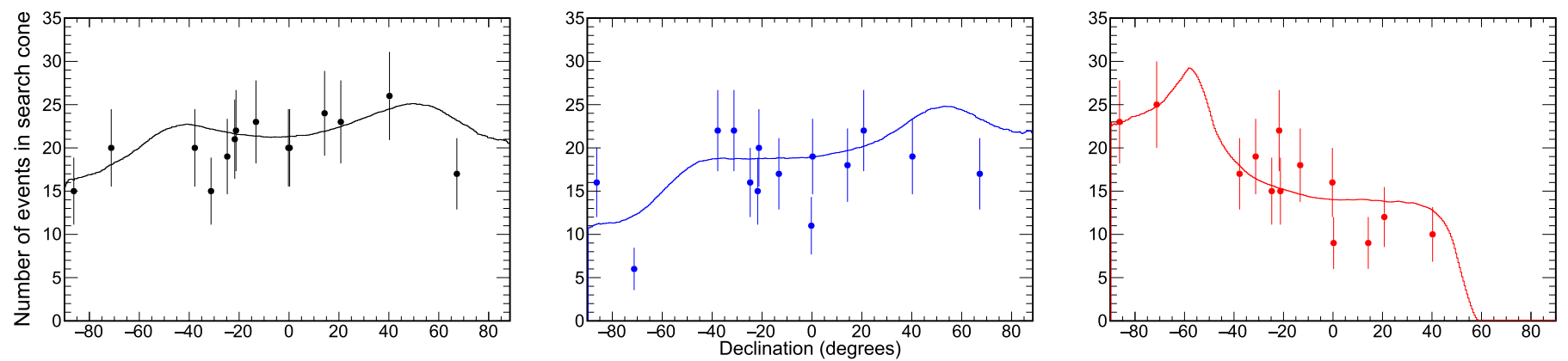

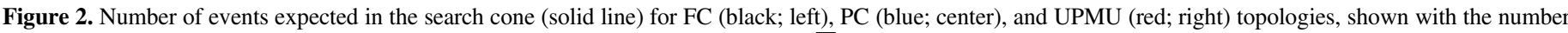

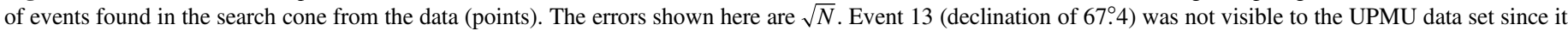
is always above the horizon in the SK detector.

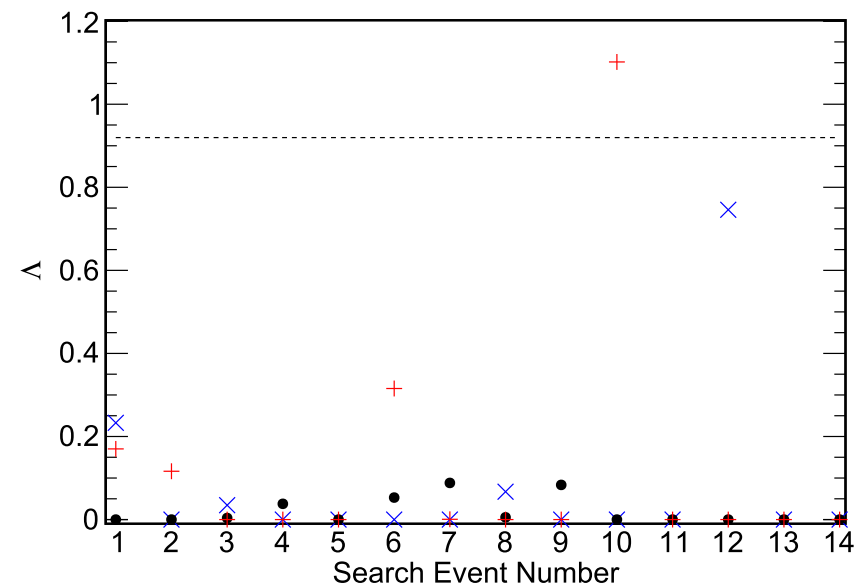

Figure 3. Test statistic for each search direction, plotted for the FC (black circle), PC (blue cross), and UPMU (red plus) topologies. The dashed line represents the $1 \sigma$ expectation calculated from the atmospheric MC. No trend can be seen for an excess in a particular search direction.

summed them together. Events were assigned a right ascension assuming a flat local sidereal time, and so the resulting $N_{B}$ was assumed to depend only on declination. The combined sample was then scaled on an event-by-event basis to the all-sky, bestfit value from data, which accounts for the flux normalization and oscillations.

Our test statistic, $\Lambda$, is then

$$
\Lambda=2 \log \frac{L\left(\alpha_{\text {fitted }}\right)}{L(\alpha=0)},
$$

where $\alpha_{\text {fitted }}$ is obtained from maximizing Equation (1). This is the final indicator of the statistical significance for the number of measured neutrino events in our search cone.

\section{Results}

Figure 1 shows the spatial distribution of the detected neutrino events in the region of each search direction. The density of the events are dependent on declination. This can be seen most clearly in the UPMU data sample, where there is a high density near the southern pole and no events at a declination above $54^{\circ}$ (see event 13 in Figure 1). The density of detected neutrino events does not appear to be significantly higher inside any of the search regions compared with the density around the search regions.

Figure 2 shows the background expectations for the three topologies considered in this search. The background expectation is assumed to be independent of the right ascension. The
FC and PC topologies extend to all declinations and have a slightly positive slope due to oscillations in the upward-going neutrinos. The two peaks are due to the atmospheric neutrinos coming from near the horizon. Here, the cosmic rays are more likely to interact due to the fact that the atmosphere is thicker and that path length for traversing this region is longer and so there is a greater chance for the cosmic ray to decay. The UPMU topology requires that the neutrino events come from below the horizon, and thus there are no events where the reconstructed direction has a declination above $54^{\circ}$. Neutrinos coming from a declination of greater than $-54^{\circ}$ spend increasingly more time above the horizon, and so there is a decreasing trend above this declination. The maximum number of UPMUs are at $-54^{\circ}$. This is because these neutrinos are near, but always below the horizon, where a greater flux of atmospheric neutrinos is expected.

We then used the SK data to calculate the number of detected events $(N)$ in the search cone for each of the IceCube search directions. Figure 2 shows the number of detected neutrino events in the search cone compared with the background expectation.

To determine if there were any statistically significant excesses in our data, we calculated the test statistic, $\Lambda$, for each search direction using Equation (2). The expected distribution of $\Lambda$ was also calculated using the 500 year simulated MC data set. The UPMU topology was used, and the declination of $-31^{\circ} .2$ was assumed. The events were first randomly assigned a right ascension assuming a flat local sidereal time. The number of events in a $5^{\circ}$ search cone was then determined, scaled for the appropriate livetime. This was compared to the background expectation $N_{B}$ for this declination, and $\Lambda$ was calculated using Equation (2). This algorithm was repeated $1 \times 10^{7}$ times, randomly assigning new right ascension values to the data each time. The expected distributions for the different topologies, as well as the different declination, were checked and found to be the same.

Figure 3 shows the test statistic for all three topologies for each search direction. $\Lambda$ was found to be zero most often, signifying that the number of events in the search cone best fit to the expected background, or that there were fewer events in the search cone than the expected background would suggest. No excess of greater than $2 \sigma$ was found for any of the topologies in any of the search directions. No search direction jointly yielded a significantly higher $\Lambda$ in all three of the topologies.

The most significant event had a calculated $\Lambda$ of 1.1 , which corresponds to a significance of about $1.1 \sigma$ from the MC background prediction. This was in the UPMU data sample in 


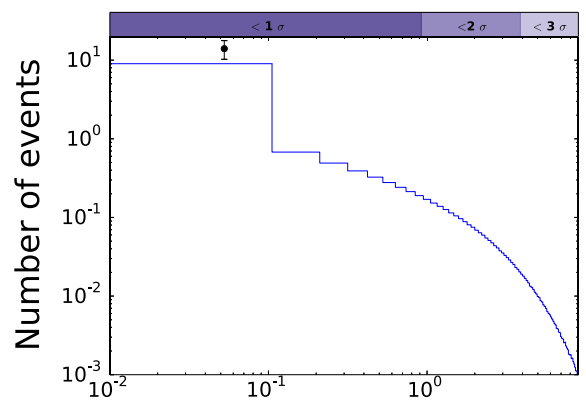

(a)

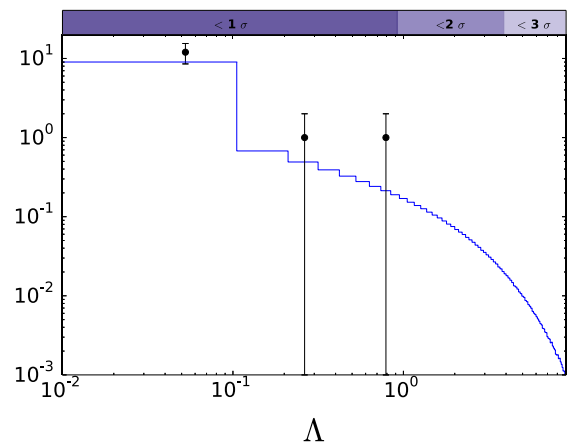

(b)

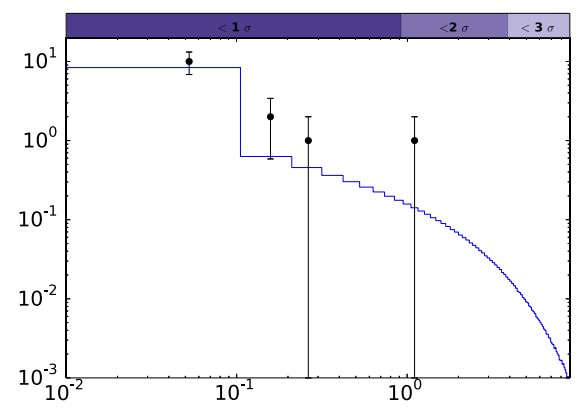

(c)

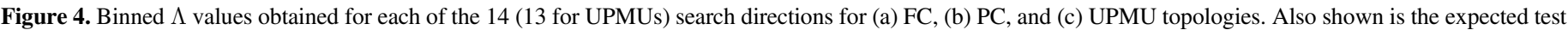

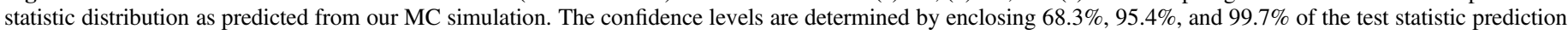
for $1 \sigma$ (dark blue), $2 \sigma$ (medium blue), and $3 \sigma$ (light blue), respectively.

the direction of event number 10, corresponding to a declination of $-22^{\circ}$. In this search direction, we observed 22 events and expected 13.7 events from the atmospheric background MC prediction.

Figure 4 shows the distribution of the test statistic separately for each of the three topologies considered, along with the expected test statistic distribution calculated using simulated data from our Monte Carlo code. As seen here, no statistically significant excesses are seen and the distribution of the test statistic from the data matches the background expectation. The confidence levels are determined using the MC test statistic distribution by calculating the $\Lambda$ where $68.3 \%, 95.4 \%$, and $99.7 \%$ of the test statistic prediction is enclosed for $1 \sigma, 2 \sigma$, and $3 \sigma$, respectively.

\subsection{Searching for a Coincidence with the IceCube Multiplet Event}

In addition to the search for excesses in the direction of the IceCube track events, we separately searched for coincidence events with the IceCube multiplet event reported in Aartsen et al. (2017). On 2016 February 17, IceCube observed three neutrino candidate events within less than $100 \mathrm{~s}$ separated by $3.6^{\circ}$. This type of multiplet event would be expected to occur once every 13.7 years. No optical counterparts were found in the follow-up searches discussed in Aartsen et al. (2017).

In SK, we searched for neutrino candidate events in the FC, PC, and UPMU data sets in a $500 \mathrm{~s}$ time window around the time of the first detected neutrino in the multiplet event. No SK candidate events were detected in any of the three topologies.

We also performed a spatial coincidence search over all SK phases using the same method used for the IceCube track events reported in the other sections of this paper. In the direction of the triplet event $\left(\mathrm{decl} .=39.5^{\circ}, \mathrm{R} . \mathrm{A} .=26.1^{\circ}\right)$, we detected (expected) 24 (24.5) FC events, 26 (23.2) PC events, and 16 (12.9) UPMU events. The likelihood ratio $(\Lambda)$ for the three topologies was calculated to be 0 for FC, 0.14 for PC, and 0.30 for UPMU, which is all less than the $1 \sigma$ value determined using the atmospheric MC background.

\section{Conclusions}

We performed a search for SK neutrino detections coincident in direction with the IceCube track events. Using Poisson statistics, we used SK data taken from 1996 April to 2016 April to determine if there was any excess of events in each of the search directions. The detected numbers of SK neutrino events in each of the search directions were consistent with the background expectations. The most significant $\Lambda$ was 1.1 at a declination of $-22^{\circ}$ in the UPMU data set, which corresponds to a significance of about $1.1 \sigma$ using the atmospheric MC prediction.

We also looked for coincidence events with the IceCube multiplet event reported in Aartsen et al. (2017). In the time coincidence search, no events were found within a $\pm 500 \mathrm{~s}$ time window from the first detected IceCube event. In the direction coincidence search, the number of events detected over the lifetime of SK from the direction of the IceCube multiplet event was consistent with atmospheric background for the FC, PC, and UPMU topologies.

These results represent the first search for coincident neutrinos with IceCube events that has been extended down to the few $\mathrm{GeV}$ energy regime. Given the unknown origin of these astrophysical neutrinos, it is worth exploring all available data in the hopes of a new discovery. This search was not optimized for a particular energy spectrum, which trades improved sensitivity to the popular energy spectra $\left(E^{-2}\right.$, for example) for the flexibility of being model independent. This search is useful for constraining the behavior of astrophysical neutrinos in the lower-energy regime and guiding new models that predict neutrino behaviors at lower energies.

We gratefully acknowledge the cooperation of the Kamioka Mining and Smelting Company. The Super-Kamiokande experiment has been built and operated from funding by the Japanese Ministry of Education, Culture, Sports, Science and Technology, the U.S. Department of Energy, and the U.S. National Science g1 Foundation. Some of us have been supported by funds from the National Research Foundation of Korea NRF20090083526 (KNRC) funded by the Ministry of Science, ICT, and Future Planning, the European Union H2020 RISEGA641540SKPLUS, the Japan Society for the Promotion of Science, the National Natural Science Foundation of China under grant No. 11235006, the National Science and Engineering Research Council (NSERC) of Canada, the Scinet and Westgrid consortia of Compute Canada, and the National Science Centre, Poland (2015/17/N/ST2/04064, 2015/18/ E/ST2/00758). 


\section{ORCID iDs}

Y. Itow (i) https://orcid.org/0000-0002-8198-1968

Y. Koshio (1) https://orcid.org/0000-0003-0437-8505

\section{References}

Aartsen, M. G., Abraham, K., Ackermann, M., et al. 2016, ApJL, 824, L28

Aartsen, M. G., Abraham, K., Ackermann, M., et al. 2017, ApJ, 835, 151

Abe, K., Haga, K., Hayato, Y., et al. 2016, ApJL, 830, L11

Abe, K., Hosaka, J., Iida, T., et al. 2006, ApJ, 652, 198

Ashie, Y., Hosaka, J., Ishihara, K., et al. 2005, PhRvD, 71, 112005

Brun, R., Bruyant, F., Maire, M., McPherson, A. C., \& Zanarini, P. 1987, CERN-DD-EE-84-1
Choi, K., Abe, K., Haga, Y., et al. 2015, PhRvL, 114, 141301

Desai, S., Abe, K., Hayato, Y., et al. 2008, APh, 29, 1

Fahey, S., Kheirandish, A., Vandenbroucke, J., \& Xu, D. 2016, arXiv:1611. 03062

Fukuda, S., Fukuda, Y., Hayakawa, T., et al. 2003, NIMA, 501, 418

Hayato, Y. 2009, AcPPB, 40, 2477

IceCube Collaboration 2013, Sci, 342, 1242856

Kadler, M., Krauß, F., Mannheim, K., et al. 2016, NatPh, 12, 807

Peng, F.-K., \& Wang, X.-Y. 2017, ApJ, 835, 269

Swanson, M. E. C., Abe, K., Hosaka, J., et al. 2006, ApJ, 652, 206

Tanaka, T., Abe, K., Hayato, Y., et al. 2011, ApJ, 742, 78

The IceCube Collaboration 2015, arXiv: 1510.05223

Thrane, E., Abe, K., Hayato, Y., et al. 2009a, ApJ, 697, 730

Thrane, E., Abe, K., Hayato, Y., et al. 2009b, ApJ, 704, 503 